\title{
Distributed Parallel Performance of Compression Molding Model for Some Characteristics Identification of Tire Tread Block.
}

\author{
Norma Alias, Roziha Darwis, Noorazura Shahira Yusniman, Nuraini Hashim
}

\begin{abstract}
A control process based on the mathematical modeling of phase change simulations was used to address process limitations of tire tread manufacturing process. The objective of this model was to predict and monitor expected characteristic identifications respect to space and time. This paper proposes a high-performance control process for visualizing the temperature behavior of a compression molding process using pressure, density, elasticity, crack propagation, volume, space, and time during tire tread block manufacturing operations. The control process modeling of tire tread blocks at the liquid-solid interface enabled the use of large sparse computations for solving the real time molding process of a Rubber Compression Molding Machine (RCMM) LWB VRE 1000 type at the Malaysia Rubber Board Research Center (LGM). The modeling simulation emphasized parallel algorithms, domain decomposition, and parallel processing techniques on Distributed Parallel Computer Architecture (DPCS). This study identified alternative numerical methods and their parallelization by comparing numerical analysis and parallel performance indicators using tables, graphs, and multidimensional visualizations. The multidimensional visualization of characteristics using the high-performance control process model accurately illustrated the heating and cooling profile of the compression molding process.
\end{abstract}

Keywords: compression molding, control process model, temperature behavior, elasticity, and high-performance analysis.

\section{INTRODUCTION}

The tire tread manufacturing process involves internal chemical mixer processes that develop sheet rubber compounds, extrusion processes for tread components, the use of high tensile martials for construction, fabric bias cutters that apply the rubber coating, component assembly, heat and pressure-based curing processes, and a final inspection using force variation and $x$-ray. In tire tread manufacturing, internal chemical reactions and processes do not fully consider

Revised Manuscript Received on October 30, 2019.

* Correspondence Author

Norma Alias*, Center for Sustainable Nanomaterials, Ibnu Sina Institute for Scientific and Industrial Research, Universiti Teknologi Malaysia, 81310 Johor Bahru, Johor, Malaysia. Email: norma@ibnusina.utm.my

Roziha Darwis, Department of Mathematical Sciences, Faculty of Science, Universiti Teknologi Malaysia, 81310 Johor Bahru, Johor, Malaysia. Email: roziha.darwis@gmail.com

Noorazura Shahira Yusniman, Department of Mathematical Sciences, Faculty of Science, Universiti Teknologi Malaysia, 81310 Johor Bahru, Johor, Malaysia. Email: noorazura.shahira@gmail.com

Nuraini Hashim, Department of Mathematical Sciences, Faculty of Science, Universiti Teknologi Malaysia, 81310 Johor Bahru, Johor, Malaysia. Email: nurainihashim36@gmail.com important characteristic identifications such as temperature profile, pressure, and elasticity, which can be used to significantly improve production processes, increase productivity, and increase product quality. Control processes based on the mathematical phase change simulation model are alternative approach to visualizing significant characteristics and making future predictions. This study proposed a high-performance control process that measured multidimensional temperature behavior such as pressure, density, elasticity, crack propagation, volume, space, and time during the compression molding process. The mathematical modeling of control processes at the liquid-solid interface enabled the use large sparse computation costs to solve the real time molding process for the Rubber Compression Molding Machine (RCMM) LWB VRE 1000 type at the Malaysia Rubber Board Research Center (LGM) (Fig. 1).

Some numerical analysis indicators and parallel performance evaluations were investigated to validate and verify the control process model for the compression molding process. This study increased the performance control process for RCMM and optimized the value-added features of tire tread molded products for the machine shown in Fig. 1.

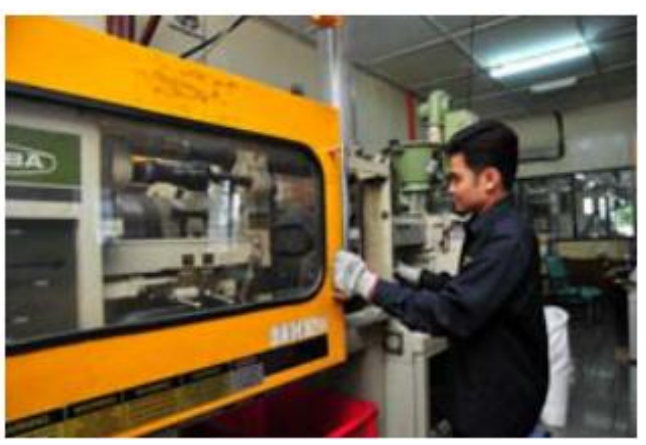

Fig. 1.Rubber compression molding machine (LWB VRE 1000) at LGM.

This paper is organized as follows: Section 2 describes the mathematical modeling and simulation used to support the thermal control process model using a phase change simulation for Stefan problems coupled as well as Navier-Stokes equations [1,2]. 


\section{Distributed Parallel Performance of Compression Molding Model for Some Characteristics Identification of Tire Tread Block.}

Section 3 introduces Finite Different Method (FDM), which uses robust methods to support the large sparse computational of model problems, the implementation sequential and parallel algorithms as well as the use of domain decomposition techniques for tire tread block development process flows. As the high-performance control process for multidimensional temperature behavior characterized many parameters, the visualization technique of the compression molding process is discussed in Section 4. The analysis briefly explains how overall complexity leads to various technical problems. Section 4 also sheds light on the Alternating Group Explicit (AGE) method and its conservation properties through numerical analysis indicators and performance evaluations [3]. Conclusions and ideas for future research are presented in Section 5.

\section{MATHEMATICAL MODELING AND SIMULATION}

Tire tread blocks are unreinforced rubber strips imbued with a specific tread pattern during molding. Treads are added as a single strip that is spliced and stitched together into several layers before being fixed directly onto the tire casing. During tread strip pre-curing, pressures between 85-110 psi are applied for four hours at $100^{\circ} \mathrm{C}$ to mold the tread. The tread pattern is applied under curing conditions (min. $140^{\circ} \mathrm{C}$ and max. $200^{\circ} \mathrm{C}$ ). Steam, hot water, or compressed air has to be maintained across the tread during curing. Process controls for temperature behaviors requires during pre and post-curing using steam, hot water, or compressed air, which are applied across the rubber tread compound. Example molding processes are compression molding, resin transfer molding, injection molding, Reaction Injection Molding (RIM), autoclave molding, and shell molding [4]. There are variations within each category as well as between mold designs, but they all meet appropriate molding process specifications at an economical rate of production [5]. The process controls of some parameters should be considered to produce a good quality molding process.

\section{A. Model Problem}

The paper focuses on the large deformation on 2D meshes. Fig. 2 illustrates the 2D cross-section design area of pre-cured tire treads during the manufacturing process with differential boundary conditions. These points occur when treads change from solid to liquid or from liquid to solid.

A phase change heat transfer simulation sequential algorithm for solid to liquid processes was used to observe the temperature profile. The mathematical model was supported by parallel $\mathrm{C}$ programming algorithms and processing architectures on a Linux operating system on Distributed Parallel Computer Architecture (DPCS).

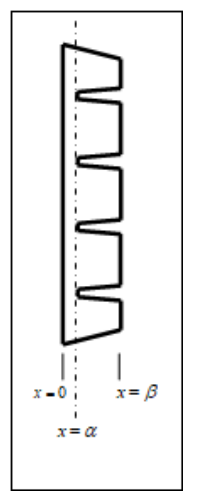

Fig. 2.2D cross section of tire tread design during the manufacturing process with boundary variables $\alpha$ and $\beta$.

\section{B. Governing Equations}

Based on the Stefan problem, the swelling of grain, polymers, and grease is governed by a nonlinear diffusion equation with two moving boundaries. This model is fundamental to creating a new model for the plasticization of amorphous and semi-crystalline rubber as well as polymer phase changes under-cooled fluids [6]. Bänsch, E. (2012) used the Stefan problem and Navier-Stokes equations for melting interface processes [7]. The FDM of the mathematical model for solid and liquid changes used Stefan conditions to identify flow fields [8]. This paper used the governing equation to improve the application of the polymer composite material model [9]. The high-performance simulations visualization of temperature behavior and crack propagation for rubber materials was analyzed using the standard techniques in [10]. This paper used phase change simulations based on the Stefan problem for $1500^{\circ} \mathrm{C}$ heating processes and $280^{\circ} \mathrm{C}$ cooling processes. A control process system for temperatures between $28-1500^{\circ} \mathrm{C}$ was applied with heating and cooling time ranges between 30-60 minutes. The aim of the thermal control process system was to maintain the rubber product at a constant length and condition.

For temperature characterization, partial differential equation (PDE) are well suited for visualization and monitoring. Stefan problems was used for the two-phase change heat transfer simulation of PDEs with parabolic type. The discretization of the two parabolic equations was based on FDM to obtain a linear system of equations that were solved numerically using iterative methods. This paper presents analytical and numerical solutions for both nonlinear and linear diffusivities with respect to changes in parameter ranges. The objective of the process model was to illustrate model dynamic properties and the limitations of using pseudo-steady-state assumptions.

Firstly, the general equation for conduction heat transfer was one-dimension with an internal cure reaction applied to each phase as given by Solomon et al. (1987):

$$
\rho_{l} C_{l} \frac{\partial T_{l}}{\partial t}=\lambda_{l} \frac{\partial^{2} T}{\partial x^{2}}+\rho_{l} \Delta T_{i, j} \quad 0<x<X_{l}(t)
$$$$
\text { (for solid to liquid) }
$$ 


$$
\rho_{s} C_{s} \frac{\partial T_{s}}{\partial t}=\lambda_{s} \frac{\partial^{2} T}{\partial x^{2}}+\rho_{s} \Delta T_{i, j} \quad x<X_{s}(t)
$$

(for liquid to solid)

where $\rho, C$, and $\lambda$ represent rubber density, rubber heat capacity, and rubber thermal conductivity, respectively. The subscripts $l$ and $s$ indicate the liquid and solid state of the rubber and $T$ is the temperature at $T(x, t)$. The contributions of conduction heat transfers and exothermic reactions is seen on the right-hand side. The heat rate evolution $\Delta T$ for the overall cure reaction at time $t$ was expressed as a function of heat evolved by time $t, T_{t}$.

The initial condition was given by:

$$
T(x, t)=T \quad T_{r}<T_{F}
$$

where $T_{r}$ is the known uniform temperature for the rubber compound before heating and $T_{F}$ is the fusion temperature at the liquid-solid interface. Since two parallel planes bound the rubber compound, the boundary conditions of the rubber were:

$$
\begin{aligned}
T(0, t)=T(\alpha, t) & =T(\beta, t)=T_{M} & & \\
-\lambda T_{s}(0, t) & =q(t) & & 0<t<t_{x} \\
T_{s}(N, t) & =0 & & 0<t<t_{x}
\end{aligned}
$$

where $T_{M}$ is the temperature of the mold, $q(t)$ is temperature changes during the phase change process, $T_{s}$ is the temperature of the liquid to solid process, and $N$ is $0 \leq x \leq N$ on the $\mathrm{x}$-axis. The equation assumed a liquid-solid interface that separated the solid region from the liquid region that was moved into the rubber over time as $X_{1}(t)$ was the location of the rubber interface at time $t, X_{1}(t)$ can be determined using the following internal energy equation:

$$
\rho_{l} L \frac{d X_{l}}{d t}=\lambda_{s} \frac{\partial T_{s}}{\partial x}-\lambda_{l} \frac{\partial T_{l}}{\partial x}
$$

where $L$ is the latent heat for melting.

Then, to solve the mathematical modelling proposed above, fully discretized (1), (2), and (5) were created for heating and cooling process using FDM. The discretization of (1), (2), and (5) are given by:

Let $r=\frac{\Delta t \lambda}{\rho C(\Delta x)^{2}}$,

$$
\text { and } T_{i, j}+1=r T_{i+1, j}+(1-2 r) T_{i, j}+r T_{i-1_{j} j}+\rho_{l} \Delta T_{i, j} \text {, }
$$

the discretization for (5) is given by:

$$
\begin{aligned}
& \text { Let, } p=\frac{\lambda_{2}}{\rho L} \text { and } q=-\left(\frac{\lambda_{l}}{\rho L}\right) \\
& X_{i, j+1}=p T_{i, j+1}-p T_{i, j}+q T_{i, j+1}+q T_{i, j}+X_{i, j} \\
& X_{i, j+1}=(p+q) T_{i, j+1}+(q-p) T_{i, j}+X_{i, j}
\end{aligned}
$$

Some numerical methods were used to simulate the phase change simulation. Using with a large sparse matrix, this paper proposed a $(2 \times 2)$ block matrix system for the group explicit method to compute the large sparse problem. The numerical methods used to solve the large sparse problem were AGE, successive over-relaxation (SOR), Red-Black Gauss-Seidel (RBGS), Gauss-Seidel (GS), and Jacobi.

\section{EXPERIMENTS}

In this study the compression molding process had 5 steps. The first step was a pre-forming or pre-heating process that blew the tread mold into the tread mold guide. Before compression, rubber 'blanks' were entered into the tread mold and cure took place for a few minutes. In the second step, the mold was closed and plasticized pre-form was used to fill the mold. Curing took place for a few minutes. In the third step, the mold was opened and final product was ejected. The press was closed and 'bumping' was used to prevent air entrapment. The rubber compound 'melted' instantly and immediately filled the mold. Pressure was applied to force the rubber compound and plastic start to take the shape of the mold. In the fourth step, the compression molding process of was completed after 13 minutes of heating. Excess rubber known as 'flash' was squeezed out from the mold cavity and removed at the end of the process. In the fifth step, the pre-cured tire tread was cooled on a brass plate. The curing reaction occurred in the heated mold and the reaction continued to take place after the rubber was removed. Fig. 3 illustrates the five steps for the compression molding and cooling processes.

Fig. 4 shows the thermal control process steps for compression molding. The general outline of the experimental process involved 7 process flows, which were tire tread block process, model governing, discretization and simulation, high performance computation, parallel programming using DPCS, visualization, monitoring, and the complete control process for the tire tread block. 
Distributed Parallel Performance of Compression Molding Model for Some Characteristics Identification of Tire Tread Block.

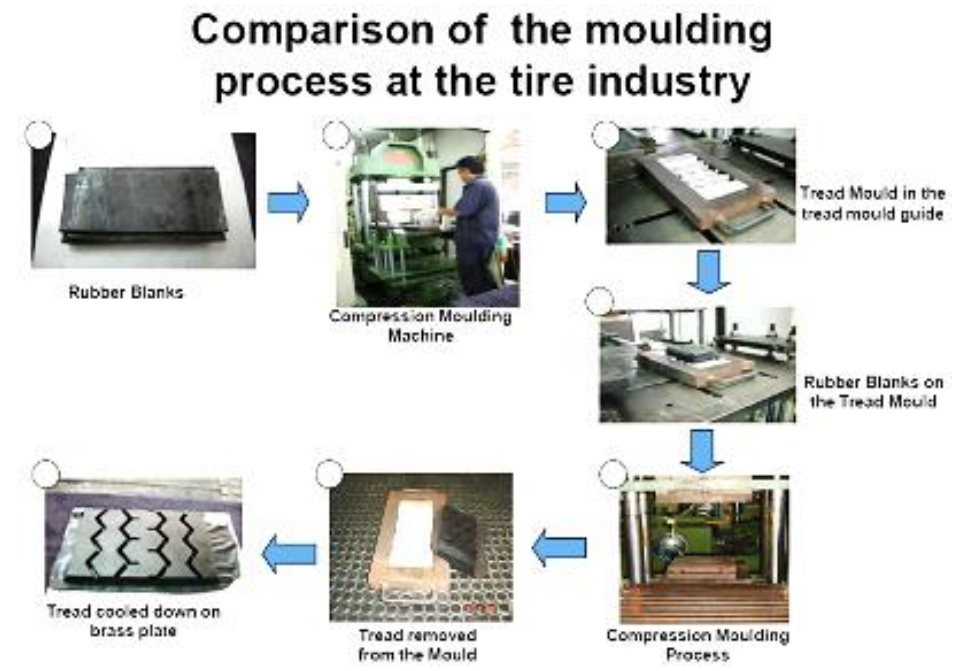

Fig. 3.Control process flow for the compression molding and cooling processes.

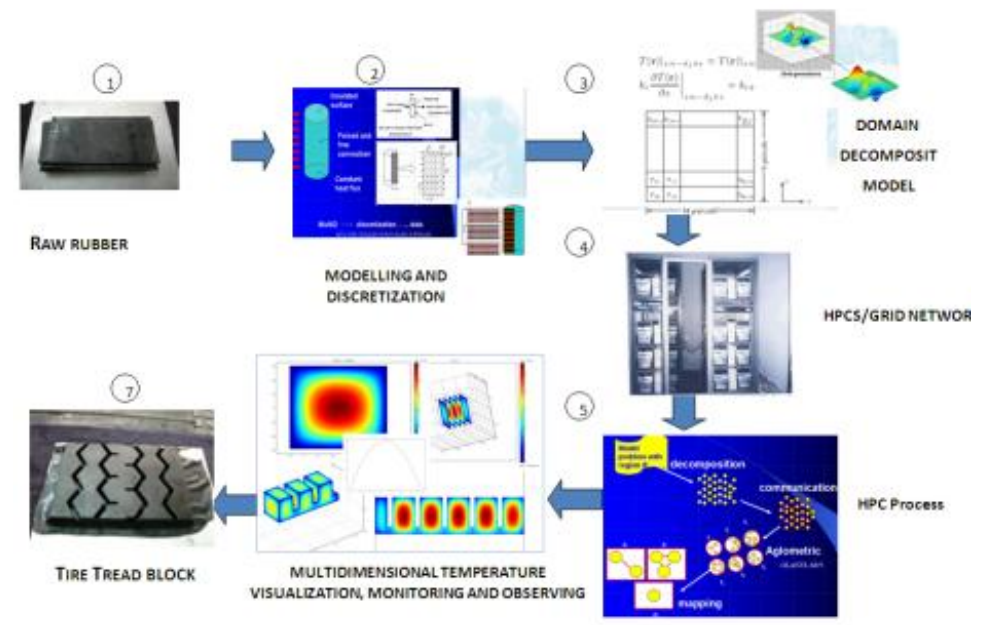

Fig. 4.Process flow for tire tread block development.

\section{NUMERICAL RESULTS AND DISCUSSION}

Discussion in this section is based on numerical analysis and parallel performance indicators. Numerical experiments were carried out on the DPCS platform using the study model to solve the Stefan problem using Navier-Stokes equations. The DPCS had an Intel Core Duo Processor, 2GB memory, and 32-bit Operating System. Matlab distributed computing and Parallel Virtual Machine (PVM) software programmed using

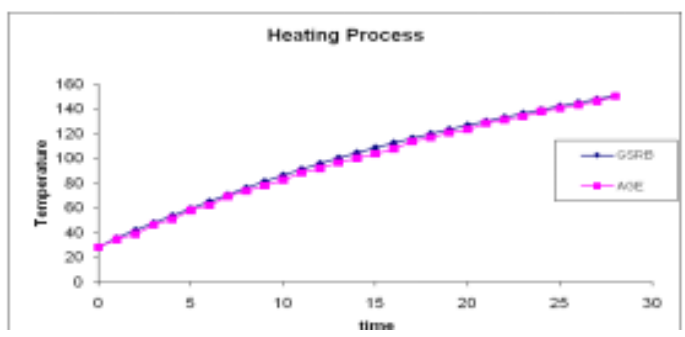

Fig. 5.Heating and curing processes for the AGE and RBGS methods.

Table 1 shows the PDE numerical results for the cooling and heating processes. The AGE model was six times more powerful than the SOR and RBGS algorithms for the convergence measures.

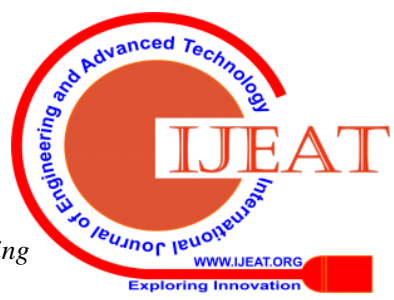


The AGE algorithm was 6, 10, and 12 times faster than SOR, RBGS, and GS, respectively. The AGE model was more predictive than SOR, RBGS, GS and Jacobi by 50\%, 56\%, $64 \%$ and $70 \%$ respectively, based on Root Mean Square Error (RMSE). AGE surpassed the approaches of RBGS, SOR, GS, and Jacobi. Depending on these findings, the contrasting quality of AGE and RBGS was the subject of the parallel development.

Table I: Numerical result for investigated numerical methods.

\begin{tabular}{|c|l|l|l|l|l|}
\hline Numerical Analysis & \multicolumn{1}{|c|}{ AGE } & \multicolumn{1}{|c|}{ RBGS } & \multicolumn{1}{|c|}{ SOR } & Jacobi \\
\hline Execution time (s) & 0.05667 & 0.06227 & 0.06228 & 0.06418 & 0.06607 \\
\hline Number of iterations & 5 & 30 & 30 & 50 & 60 \\
\hline RMSE & 1.77057 & 4.03773 & 3.56559 & 4.93908 & 5.79083 \\
\hline Convergence rate & 12 & 2 & 2 & 1.2 & 1 \\
\hline
\end{tabular}

The multidimensional visualization of compression molding was influenced by density, pressure, elasticity, crack propagation, and multidimensional temperature behavior as shown in Fig. 6. Fig. 6(a) illustrates density increases and refers to the relationship between mass and volume. According to Fig. 6(b), there was an optimum level of pressure and strong elasticity at the corners of two-dimensional tire tread. Fig. 6(d) shows potential crack propagation areas and insufficient crack resistance. The multidimensional temperature behavior of the molding process is visualized in Fig. 6(e) and (f). Inside the tread block, the temperature was significantly higher than outside the tire block.

a) $1 \mathrm{D}$ and $2 \mathrm{D}$ density.

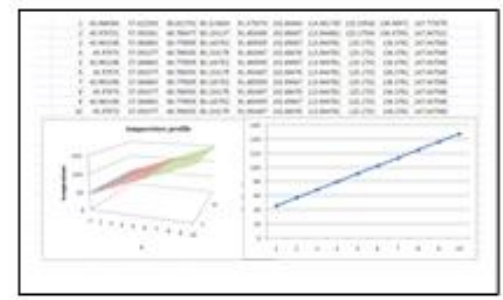

b) $1 \mathrm{D}$ pressure.

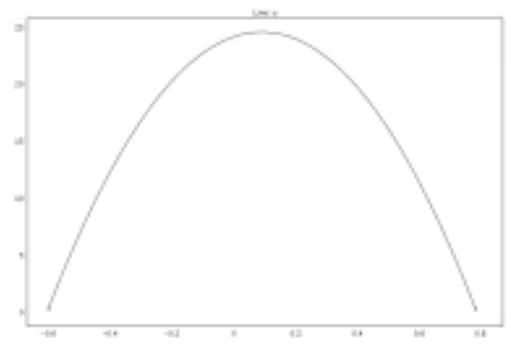

c) Elasticity.

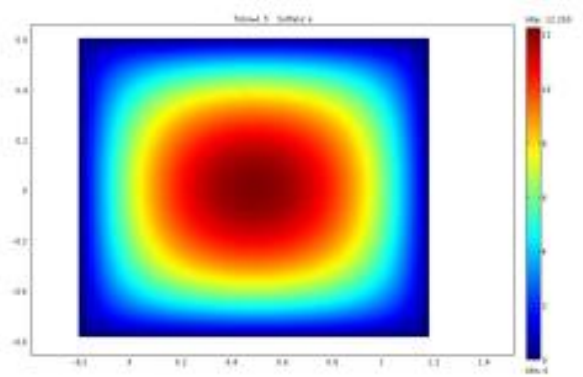

d) $3 \mathrm{D}$ crack propagation.

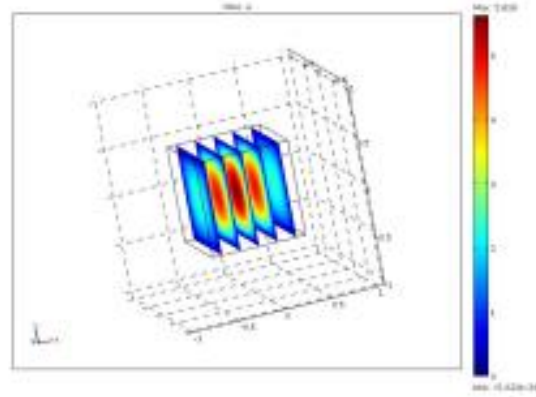

e) $2 \mathrm{D}$ temperature behavior.

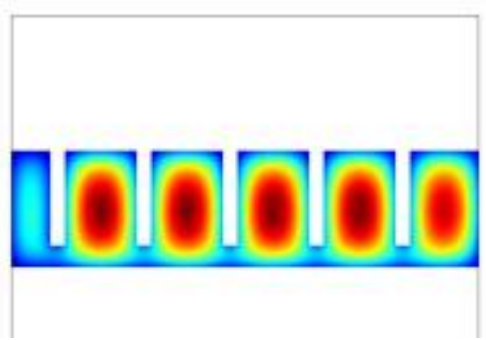

f) 3D temperature behavior with respect to space and time.

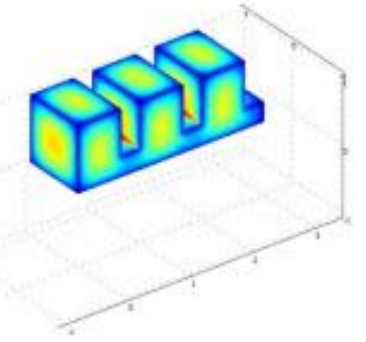

Fig. 6. Multidimensional visualization of compression molding.

A comparison of the governing model's cure percentages with the actual figures obtained at LGM can be seen in the Fig. 7. The outcome indicates that at every stage of the period, the theoretical formula (EPCL) translated to the real value curve. It has been shown that the governing approach for the compression molding system has made fairly smart predictions over long time measures.

Published By:

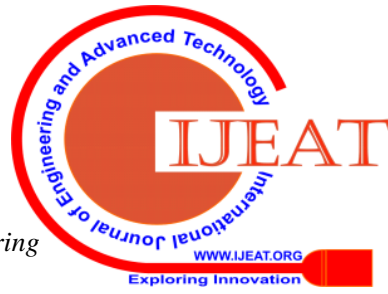




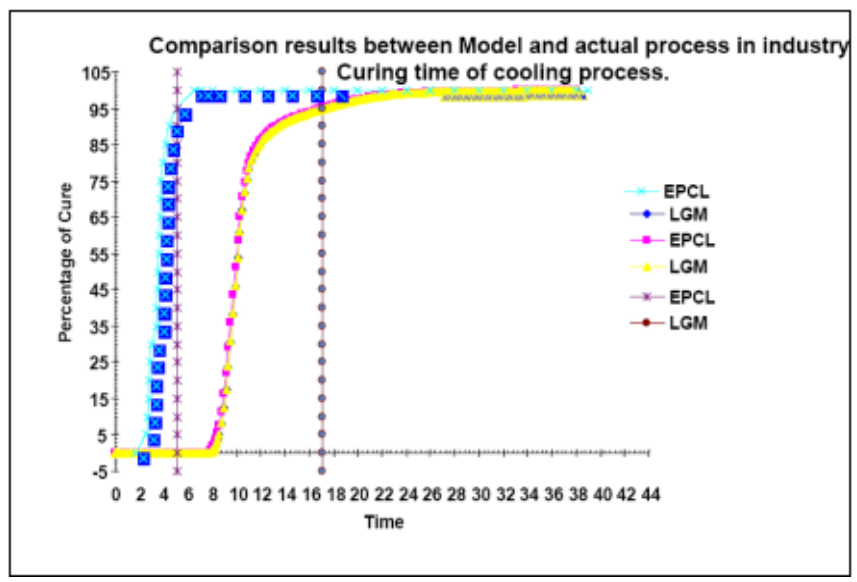

Fig. 7.Comparison results between Model and real time data set at LGM for compression molding process.

\section{B. Parallel Performance Evaluations}

Fig. 8 shows the parallel performance comparison for AGE and RBGS. Fig. 8(a) shows that execution time decreased with and increased number of processors (p). The speed and efficiency of the AGE method showed a greater increase with increased processor number compared to RBGS as shown in in Fig. 8(b) and (c). The potential of parallel algorithms, domain decomposition techniques, task distribution over memory space, and communication frequency are determined by optimum effective parallelism. Thus, Fig. 8(d) and (e) show the significantly greater effectiveness and temporal performance of AGE over RBGS method. (a)

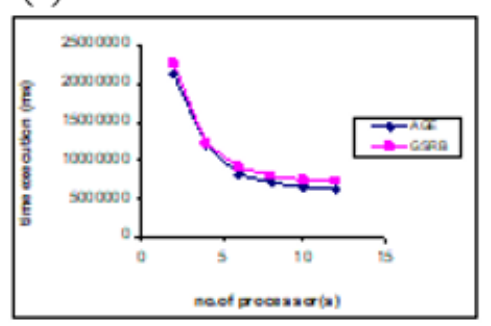

(d)

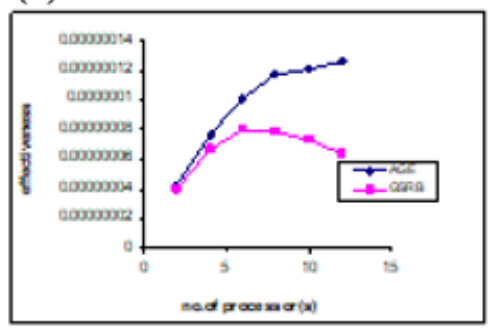

(b)

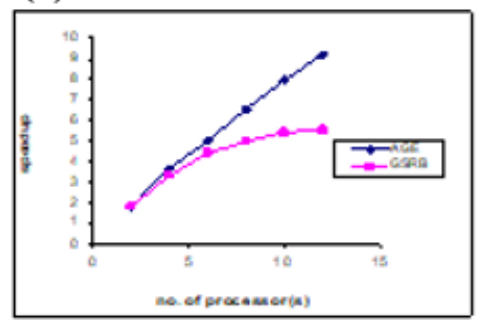

(e)

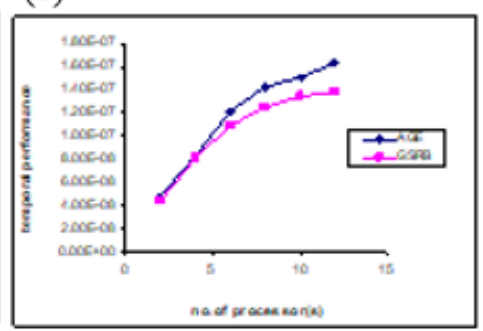

(c)

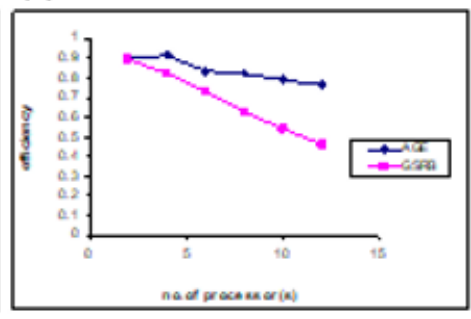

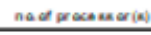

y; (d) effectiveness; and (e) temporal performance versus number of processors

\section{CONCLUSIONS AND IDEAS FOR FUTURE RESEARCH}

Based on the numerical and parallel experiment results, this paper concludes that the improved Stefan problem coupled with the Navier-Stokes equations made two-dimensional diffusion PDEs created a compression molding process with improved efficiency, speed, and accuracy. The improved Stefan problem coupled with the Navier-Stokes equations visualized the compression molding process and identified the influence of pressure, density, elasticity, crack propagation respect to volume, space, and time. The obtained solutions, numerical analysis, and parallel performance of specific indicators were used to analyze the heating and cooling profile of the compression molding process. Furthermore, the AGE method was efficient and well suited to finding exact solutions for real time molding processes that use large sparse computation for some parameters.

In conclusion, the proposed models' tables, graphs, multidimensional visualization, and numerical validation show the efficient prediction of compression molding processes. The parallel code repository was optimized to acquire a computation speedup of approximately 50\% for sequential computational costs. This study's model is well suited to integrating numerical repositories and embedded system software for RCMM operations at LGM.

\section{ACKNOWLEDGMENT}

This research was supported by Malaysia Government under the UTM TIER 1 grant code PY/2014/02324.

\section{REFERENCES}

1. M. C. Olguin, M. A. Medina, M.C. Sanziel, and D.A. Tarzia, "Behavior of the solution of a Stefan problem by changing thermal coefficients of the substance," Applied Mathematics And Computation, 190(1), 2007, pp. 765-780.

2. A. Solomon, V. Alexiades, and D. G. Wilson, "The initial velocity of the emerging free boundary in a two-phase Stefan Problem with imposed flux," SIAM journal on mathematical analysis, 18(5), 1987, pp. 1438-1452.

3. N. Alias, M. S. Shimi, A. R. Abdullah, "The AGEB Algoritma for Solving the Heat Equation in Two Space Dimensions and Its Parallelization on a Distributed Memory Machine," SpringerLink, vol. 2840, 2003, pp. 214-221.

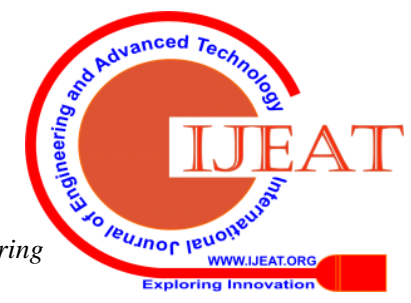


4. P. Sae-oui, and U. Thepsuwan, "Prediction of cure level in thick rubber cylinder using finite element analysis," Science Asia, 28, 2002, pp. 385-391.

5. J. P. Pascault, H. Sautereau, J. Verdu, and R. J. J. Williams, "Thermosetting polymers," Vol. 64, CRC press, New York: Macel Dekker, Inc., 2002.

6. G. Zitzenbacher, and G. R. Langecker, "A new plasticating model for amorphous and semi-crystalline polymers in single screw extruders," International Polymer Processing, 20 (4), 2005, pp. 360-368.

7. E. Bänsch, J. Paul, and A. Schmidt, "An ALE finite element method for a coupled Stefan problem and Navier-Stokes equations with free capillary surface." International Journal for Numerical Methods in Fluids 71(10), 2013, pp. 1282-1296.

8. N. Alias, R. Darwis, N. Satam, and M. Othman, "Parallelization of temperature distribution simulations for semiconductor and polymer composite material on distributed memory architecture," In International Conference on Parallel Computing Technologies, pp. 392-398. Springer, Berlin, Heidelberg, 2009, pp. 393-398.

9. R. Darwis, N. Alias, N. Yaacob, M. Z. Othman, N. Abdullah, and Y. Y The, "Temperature behavior visualization on rubber material involving phase change simulation," Malaysian Journal of Fundamental and Applied Sciences, 5(1), 2009.

10. N. Alias, S. T. Yin and R. Darwis and I. Rajibul, "Finite element of crack propagation discretization and its parallelization on distributed parallel computing systems," In: 5th Asian Mathematical Conference (AMC 2009), Pusat Dagangan Dunia Putra (PWTC), Kuala Lumpur, 2009.

\section{AUTHORS PROFILE}

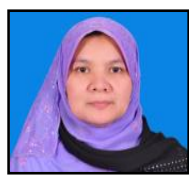

Norma Alias is an Associate Professor of Industrial and Parallel Computing, Research Fellow and Head of Network Division and International Relations, Ibnu Sina Institute for Fundamental Science Studies (IIS) at Universiti Teknologi Malaysia. In 2004, she holds a PhD in Industrial Computing (Parallel Computing) from Universiti Kebangsaan Malaysia. She has published over 200 publications. She also obtained 2 patents and 2 products for commercialization, 4 intellectual property declaration, 16 Innovation and Invention Medal since 2004. She has various areas of expertise including mathematical modeling, big data simulation, and parallel programming by using the high-performance computation and software development.

Roziha Darwis holds a Master of Philosophy in Mathematics in 2011 from Universiti Teknologi Malaysia. Her research work focuses on parallel programming. She has published 13 publications, 6 of them are Scopus-indexed publications.

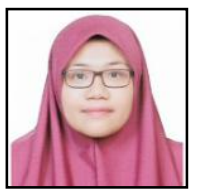

Noorazura Shahira Yusniman is currently undergoing a postgraduate study in the field of Mathematics at Universiti Teknologi Malaysia. Her main research works focuses on EEG signal of eye movements and the relations with stress level. Using machine learning and parallel programming, the predictions of stress are being investigated. In 2017, she received her Bachelor Degree in Mathematics. Her research interest includes big data simulation, machine learning, parallel programming, and data science.

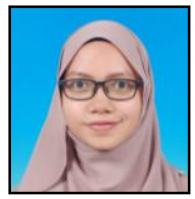

Third Author is currently undergoing a study in the field of Mathematics at Universiti Teknologi Malaysia. Her main research focuses on modelling of drug delivery using high performance computing. In 2017, she received her Bachelor Degree in Mathematics at the same university. Her research intereste includes mathematical modelling, drug delivery, big data simulation and high-performance computing. 